\title{
Saberes e práticas: ações de promoção ao aleitamento materno desenvolvidas pela equipe de enfermagem: revisão integrativa
}

\author{
Knowledge and practices: actions to promote breastfeeding developed by the nursing team: \\ integrative review \\ Conocimientos y prácticas: acciones para promover la lactancia materna desarrolladas por el \\ equipo de enfermería: revisión integrativa
}

Deborah Larissa Souza de Santos ORCID: https://orcid.org/0000-0001-7679-6648 Universidade Ceuma, Brasil E-mail: deeborahlarissa@gmail.com

Benedita Maryjose Gleyk Gomes ORCID: https://orcid.org/0000-0002-5231-3936 Universidade Ceuma, Brasil E-mail: bene.belo@outlook.com

Haigle Reckziegel de Sousa ORCID: https://orcid.org/0000-0002-5803-2289 Universidade Ceuma, Brasil E-mail: haiglereck3@gmail.com Aline de Sousa Rocha ORCID: https://orcid.org/0000-0002-8181-0835 Universidade Ceuma, Brasil E-mail: lillydarocha@hotmail.com

Maksandra Silva Dutra ORCID: https://orcid.org/0000-0003-0809-6097 Universidade Ceuma, Brasil E-mail: maksandrad@hotmail.com

Marluce Sampaio Nobre Barbosa ORCID: https://orcid.org/0000-0001-7276-6521 Universidade Ceuma, Brasil E-mail: marluce.nobre@ceuma.br

Francisco Alves Lima Júnior ORCID: https://orcid.org/0000-0002-3117-4949 Universidade Ceuma, Brasil

E-mail: francisco.enfdotrabalho@gmail.com

\begin{abstract}
Resumo
Introdução: $\mathrm{O}$ incentivo ao aleitamento materno (AM), corresponde a uma estratégia de grande necessidade, ao mesmo tempo em que torna natural o vínculo, afeto, proteção e nutrição da criança. Objetivo: O objetivo geral é conceituar as ações de promoção ao aleitamento materno desenvolvidas pela equipe de enfermagem. Metodologia: Revisão integrativa, de abordagem qualitativa, de caráter exploratório. Resultados: $\mathrm{O}$ enfermeiro deve identificar durante o pré-natal os conhecimentos, a experiência prática, as crenças e a vivência social e familiar da gestante com a finalidade de promover educação em saúde para o aleitamento materno, assim como, garantir vigilância e efetividade durante a assistência a nutriz no pós-parto. Considerações finais: Observou-se que a enfermagem tem grande contribuição quando se trata do apoio para a prática do aleitamento materno e a conscientização sobre o desmame precoce, pois tem conhecimentos práticos e teóricos para oportunizar momentos educativos, orienta gestante e puérperas sobre a importância do Aleitamento Materno e o manejo correto da lactação.
\end{abstract}

Palavras-chave: Aleitamento materno, Enfermagem, Promoção da saúde.

\begin{abstract}
Introduction: The encouragement of breast-feeding (AM), corresponds to a strategy of great need, while making the link, affection, protection and nutrition of the child natural. Objective: The general objective is to conceptualize the breast-feeding promotion actions developed by the nursing team.Methodology: Integrative review, with a qualitative approach, of an exploratory nature.Results: The nurse should identify during prenatal care the pregnancy's knowledge, practical experience, beliefs and social and family experience with the aim of promoting health education for breastfeeding, as well as ensuring vigilance and effectiveness during postpartum nutrition care.Final considerations: Nursing has made a major contribution when it comes to supporting the practice of breast-feeding and raising
\end{abstract}


awareness of early weaning, because it has practical and theoretical knowledge to opportune educational moments,It guides pregnant women and workers on the importance of breast-feeding and the correct management of lactation.

Keywords: Breast feeding, Nursing, Health promotion.

\section{Resumen}

Introducción: el fomento de la lactancia materna (AM) es una estrategia muy necesaria para hacer que los vínculos, las emociones, la protección y la nutrición de los niños sean naturales. Objetivo: el objetivo general es conceptualizar las acciones de promoción de la lactancia materna establecidas por el equipo de enfermería. Métodos: se utilizaron métodos cualitativos exploratorios para realizar un examen amplio. Resultados: durante la atención prenatal, los enfermeros deben determinar los conocimientos, la experiencia práctica, las creencias y la experiencia social y familiar de las mujeres embarazadas para promover la educación sanitaria en la lactancia materna y garantizar la vigilancia y eficacia de la atención nutricional posparto. Consideraciones finales: la enfermería ha hecho una gran contribución al apoyo a la práctica de la lactancia materna y a la sensibilización sobre el destete precoz, ya que la enfermería tiene conocimientos prácticos y teóricos que permiten una educación oportuna. Guía a las mujeres embarazadas y a los trabajadores sobre la importancia de la lactancia materna y el manejo adecuado de la lactancia.

Palablas clave: Lactancia materna, Enfermería, Promoción de la salud.

\section{Introdução}

Todas as crianças devem ser amamentadas de forma exclusiva até os 6 meses de vida e, após esse período, deve ser iniciada a alimentação complementar, mantendo-se a amamentação até pelo menos os 2 anos de idade. O desmame precoce é o resultado de uma interrupção do aleitamento materno ao peito antes de o lactente ter completado o que se chama de idade adequada, ou seja, seis meses de vida, independentemente da decisão ser da mãe ou não e do motivo da interrupção. na maioria das vezes, as crenças da família e a cultura influenciam diretamente tanto nos métodos de amamentação como na interrupção (Wilhelm et al.,2015) (Oliveira et al.,2015).

Quando ocorre a desistência da amamentação ou intervalo, existe a troca e/ou escolha por outros tipos de alimentos na dieta da criança, trazendo consequências potencialmente danosas à saúde do bebê, entre elas, a exposição a agentes infecciosos, contato com proteínas estranhas e prejuízos ao processo de digestão. Com o desmame precoce, a mãe também elimina a proteção natural contra a contracepção e o câncer da mama e do ovário (Rocha et al., 2016).

Entre as ações de maior importância utilizadas pelo enfermeiro na consulta à criança, destaca-se o incentivo ao aleitamento materno (AM), corresponde a uma estratégia de grande necessidade, ao mesmo tempo em que torna natural o vínculo, afeto, proteção e nutrição da criança. Constitui a mais sensível, econômica e eficaz intervenção para a redução da mortalidade infantil, definida pelas políticas públicas, especialmente pela Agenda de Compromissos para Atenção Integral à Saúde da Criança e Redução da Mortalidade Infantil (Monteschio, Gaíva \& Moreira, 2015). No entanto, mesmo com a existência de políticas e investimentos para melhorar os níveis de aleitamento materno exclusivo no Brasil, o país ainda está distante de conseguir obedecer às recomendações da Organização Mundial da Saúde (OMS) (Monteschio, Gaíva \& Moreira, 2015).

Os resultados de pesquisas bibliográficas sempre destacam que as ações de enfermagem educativas e os cumprimentos protocolares no que concerne o cuidado da gestante e do bebê propiciaram experiências benéficas para ambos. No cotidiano assistencial, os enfermeiros encontram várias puérperas, e assim, encontram as chamadas "mães de primeira viagem", que iniciam a amamentação, mas sempre se queixam de dificuldades. Entretanto, algumas precisam de apoio, incentivo e principalmente de um trabalho de orientação, pois ainda não se sentem seguras diante do novo desafio de nutrir um ser humano tão pequeno e frágil (Amaral et al., 2015).

Independentemente dos diversos benefícios que o aleitamento materno propicia e dos inúmeros programas de incentivo ao aleitamento materno exclusivo até os primeiros seis meses de vida do recém-nascido, o desmame precoce ainda prevalece principalmente nas classes sociais menos favorecidas, trazendo prejuízos tanto para o bebê como para mãe e também 
para a família, além de tornar um problema de saúde pública, sendo assim, o objetivo geral é conceituar as ações de promoção ao aleitamento materno desenvolvidas pela equipe de enfermagem.

\section{Metodologia}

A presente pesquisa é baseada na revisão integrativa, aquela que visa contextualizar um problema científico de forma sistemática, para exercer a aplicabilidade do tema, tendo esse nome pois promove resultados mais amplos do assunto, trazendo um corpo de conhecimento, integrando diferentes finalidades, direcionado aos vários conceitos, teorias ou métodos (Paiva et al.,2016) (Galvão, Pansani \& Harrad, 2015).

Além de ser uma revisão integrativa, tem abordagem qualitativa, de caráter exploratório, sendo assim, a pesquisa qualitativa é aquela que são visadas opiniões sobre o estudo, de classificação exploratória, para que o problema estudado seja melhor representando, trazendo clarezas as hipóteses encontradas (Fantinato et al.,2015).

As etapas constituídas são: questão norteadora, artigos na literatura nacional, busca dos artigos a partir de critérios de inclusão e exclusão, avaliação, análise e discussão dos resultados.

A questão norteadora é: quais as ações de promoção ao aleitamento materno desenvolvidas pela equipe de enfermagem?

Os dados inclusão foram artigos de janeiro de 2016 a setembro de 2021, com os Descritores Aleitamento Materno, Enfermagem e Promoção da Saúde, sendo elencados pelo Descritores em Saúde (DECS). Os critérios de exclusão denotaram todos os artigos anteriores ao ano de 2016, Trabalhos de Conclusão de Curso (TCCs), monografias, dissertações, teses, relatos de caso e experiência, além de estudos que estiverem condizentes com o estudo.

Para a resposta da pergunta, utilizou-se da estratégia de PICO, que utilizada deste acrônimo para contextualizar as seguintes questões: P) - a população a ser estudada; (I) - interesse do assunto; (C) - controle da pesquisa, dados de inclusão e exclusão (O) - são os resultados da pesquisa (Ribeiro, Martins \& Tronchin, 2015), conforme o Quadro 1.

Quadro 1. PICO utilizado no estudo.

\begin{tabular}{|l|l|}
\hline \multicolumn{1}{|c|}{ ACRÔNIMO } & \multicolumn{1}{c|}{ DEMOSTRAÇÃO } \\
\hline $\mathrm{P}$ & Equipe de enfermagem \\
\hline $\mathrm{I}$ & Reunir os estudos acerca das ações de promoção ao aleitamento materno pela equipe de enfermagem \\
\hline $\mathrm{C}$ & Conceituar as ações de enfermagem de promoção ao aleitamento materno pela equipe de enfermagem \\
\hline $\mathrm{O}$ & As ações de enfermagem de promoção ao aleitamento materno pela equipe de enfermagem \\
\hline
\end{tabular}

Fonte: Autores (2021).

As bases de dados escolhidas foram Scientific Electronic Library Online (SCIELO), Literatura Latino-Americana e do Caribe em Ciência da Saúde (LILACS) e BIREME, utilizado os descritores supracitados, na língua portuguesa e inglesa, combinados entre si pelo termo boleano AND e na utilização de sinônimos empregou-se OR, encontrou-se Aleitamento Materno (n= (389)), Promoção da Saúde (231) e Papel do Profissional de Enfermagem (162). Utilizou-se a leitura dos resumos como ponto de referência inicial para a seleção dos artigos, para identificar, desta forma, o tema da pesquisa e se estavam dentro dos parâmetros de inclusão. A escolha final dos artigos ocorreu por meio da leitura, na íntegra, dos trabalhos previamente selecionados. A coleta de dados se baseou no formulário, abordando observações como título, primeiro autor, ano de publicação, tipo de estudo, base e conclusão, sendo todos organizados no formato de quadro. 
Inicialmente, selecionou-se 300 artigos dentro dos padrões estudados, após isso, somente 189 foram para seleção final a meio da leitura detalhada e avaliação dos critérios previamente estabelecidos, foi possível elencar 10 artigos para a construção do trabalho final. De acordo com fluxograma PRISMA (Salvador et al., 2020):

Figura 1. Fluxograma do processo de seleção dos artigos da revisão integrativa, 2021.

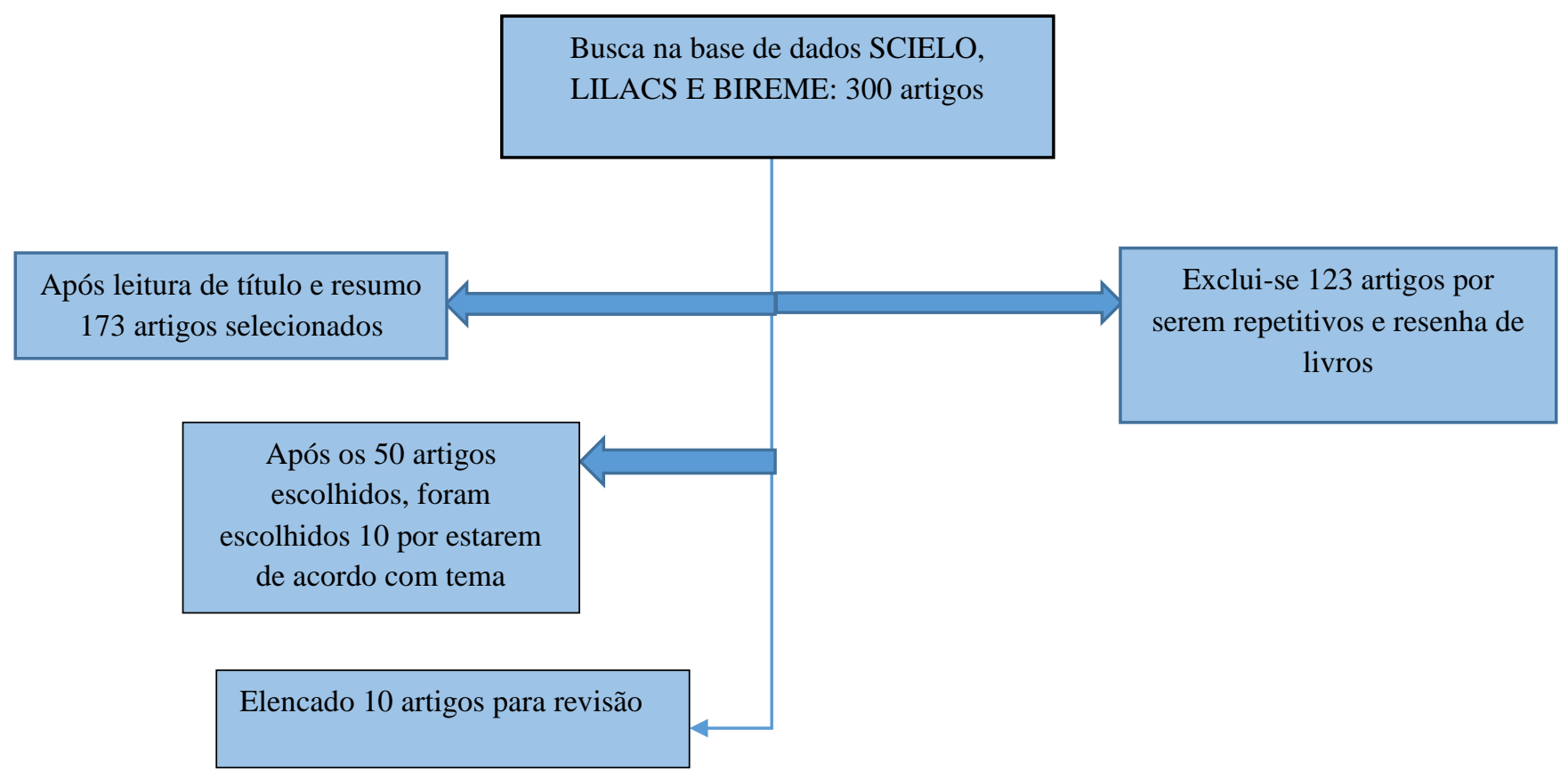

Fonte: Autores (2021).

Desta forma, os artigos selecionados por meio da estratégia de busca foram inicialmente filtrados pela leitura dos títulos. Em um segundo momento, os resumos foram lidos para identificar se estavam adequados e se possuíam temas pertinentes ao trabalho. Os artigos escolhidos através da segunda etapa, foram lidos por completo a fim de selecionar o material de análise.

\section{Resultados}

Inicialmente, foram identificados 300 artigos, sendo estes submetido à seleção dos filtros de busca, de acordo com os critérios de inclusão previamente estabelecidos. Desta filtragem inicial, sobraram 173 artigos eleitos para a avaliação dos títulos e resumos. Após a leitura, 123 artigos foram eliminados por não corresponderem aos estudos em questão, sobraram então 50 estudos para parecer. Compuseram o presente trabalho 10 artigos, resultantes do processo de filtragem, de acordo com Quadro 2: 
Quadro 2. Artigos incluídos na revisão de acordo com ano, primeiro autor, título, tipo de estudo e conclusões.

\begin{tabular}{|c|c|c|c|c|}
\hline ANO & $\begin{array}{c}\text { NOME DO } \\
\text { PRIMEIRO AUTOR }\end{array}$ & TÍTULO & TIPO DE ESTUDO & CONCLUSÃO \\
\hline 2021 & $\begin{array}{l}\text { Andressa de Oliveira } \\
\text { Rios Pereira }\end{array}$ & $\begin{array}{l}\text { Fatores que interferem } \\
\text { na realização do } \\
\text { aleitamento materno } \\
\text { exclusivo }\end{array}$ & Revisão Sistemática. & $\begin{array}{l}\text { O êxito do AME se faz com uma } \\
\text { abordagem holística } \\
\begin{array}{l}\text { dos profissionais de Enfermagem. } \\
\text { efetiva }\end{array}\end{array}$ \\
\hline 2021 & $\begin{array}{l}\text { Anna Beatriz Lira da } \\
\text { Silva }\end{array}$ & $\begin{array}{l}\text { Experiência e atitudes } \\
\text { de gestantes acerca do } \\
\text { aleitamento materno }\end{array}$ & $\begin{array}{l}\text { Estudo qualitativo } \\
\text { mediatizado por } \\
\text { uma pesquisa-ação. }\end{array}$ & $\begin{array}{l}\text { Observaram-se as lacunas existentes } \\
\text { no conhecimento das gestantes investigad } \\
\text { as sobre o aleitamento materno no que se } \\
\text { refere aos benefícios maternos da prática } \\
\text { em questão. }\end{array}$ \\
\hline 2021 & $\begin{array}{l}\text { Giovana Callegaro } \\
\text { Higashi }\end{array}$ & $\begin{array}{c}\text { Práticas de } \\
\text { enfermeiros e a } \\
\text { influência } \\
\text { sociocultural na } \\
\text { adesão ao aleitamento } \\
\text { materno }\end{array}$ & $\begin{array}{l}\text { Método estudo } \\
\text { qualitativo } \\
\text { à luz da teoria } \\
\text { fundamentada nos } \\
\text { dados. }\end{array}$ & $\begin{array}{l}\text { Os enfermeiros apontaram inúmeras } \\
\text { práticas para o fortalecimento e a adesão } \\
\text { ao aleitamento materno desde o pré- } \\
\text { natal ao puerpério. }\end{array}$ \\
\hline 2021 & $\begin{array}{l}\text { Marina Delli Zotti } \\
\text { Souza Viana }\end{array}$ & $\begin{array}{l}\text { Estratégias e ações do } \\
\text { enfermeiro no } \\
\text { incentivo ao } \\
\text { aleitamento materno: } \\
\text { revisão integrativa }\end{array}$ & Revisão integrativa. & 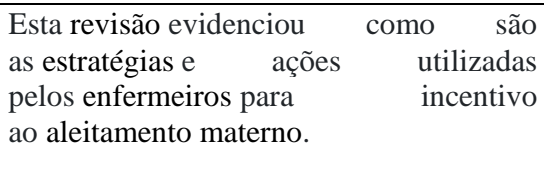 \\
\hline 2020 & $\begin{array}{l}\text { Ilva, Luana Santiago da } \\
\text { Silva }\end{array}$ & $\begin{array}{l}\text { Contribuição do } \\
\text { enfermeiro ao } \\
\text { aleitamento materno } \\
\text { na atenção básica } \\
\end{array}$ & $\begin{array}{l}\text { Trata-se de um estudo } \\
\text { exploratório e } \\
\text { descritivo, com } \\
\text { abordagem qualitativa. }\end{array}$ & $\begin{array}{l}\text { O enfermeiro tem papel essencial na } \\
\text { orientação sobre ao aleitamento } \\
\text { materno na atenção básica. }\end{array}$ \\
\hline 2020 & $\begin{array}{l}\text { Suellen Cristina dias } \\
\text { Emidio }\end{array}$ & $\begin{array}{l}\text { Revisão dos } \\
\text { indicadores para os } \\
\text { resultados de } \\
\text { enfermagem } \\
\text { relacionados ao } \\
\text { estabelecimento da } \\
\text { amamentação }\end{array}$ & Revisão de literatura. & $\begin{array}{l}\text { Indicadores podem orientar a avaliação } \\
\text { da amamentação de forma contínua, bem } \\
\text { como da efetividade das intervenções, } \\
\text { além de tornarem o registro padronizado } \\
\text { e poderem auxiliar o processo educativo } \\
\text { de estudantes e enfermeiros. }\end{array}$ \\
\hline 2020 & Evaldo Lustosa & $\begin{array}{l}\text { Importância da } \\
\text { enfermagem frente à } \\
\text { assistência primária ao } \\
\text { aleitamento materno } \\
\text { exclusivo na atenção } \\
\text { básica }\end{array}$ & Revisão Integrativa. & $\begin{array}{l}\text { O papel da enfermagem é garantir } \\
\text { através da promoção, proteção e } \\
\text { prevenção a prática do AME, não só } \\
\text { através da informação, mas } \\
\text { principalmente pela implementação de } \\
\text { ações. }\end{array}$ \\
\hline 2016 & $\begin{array}{l}\text { Ariele Londres } \\
\text { Mesquita }\end{array}$ & $\begin{array}{c}\text { Atribuições de } \\
\text { enfermeiros na } \\
\text { orientação de } \\
\text { lactantes acerca do } \\
\text { aleitamento materno }\end{array}$ & Revisão de Literatura. & $\begin{array}{l}\text { Enfermeiro tem um papel importante na } \\
\text { prevenção e promoção de saúde a estas } \\
\text { nutrizes, estratégias devem ser criadas em } \\
\text { conjunto com equipes de saúde para } \\
\text { diminuir o índice de intercorrências } \\
\text { mamarias e consequentemente garantir } \\
\text { um período de amamentação adequado } \\
\text { sem prejuízos tanto para a mãe quanto } \\
\text { para o bebê. }\end{array}$ \\
\hline 2016 & Caetano da Cunha & $\begin{array}{c}\text { Aleitamento } \\
\text { materno: } \\
\text { contribuições da } \\
\text { enfermagem }\end{array}$ & Revisão de Literatura. & $\begin{array}{l}\text { As pesquisas assinalam a importância do } \\
\text { preparo da enfermagem na atuação no } \\
\text { aleitamento materno e a consideram como } \\
\text { sendo fundamental, quer seja na } \\
\text { assistência hospitalar como nas unidades } \\
\text { básicas. }\end{array}$ \\
\hline 2019 & Luzia Fabiana de Sousa & $\begin{array}{c}\text { Desafios e } \\
\text { potencialidades na } \\
\text { assistência de } \\
\text { enfermagem no } \\
\text { aleitamento materno }\end{array}$ & Revisão de Literatura. & $\begin{array}{l}\text { A enfermagem por ter maior contato com } \\
\text { a mulher em seu período gravídico } \\
\text { puerperal, o vínculo entre ambos se torna } \\
\text { uma das principais potencialidades para } \\
\text { efetivação da amamentação, fortalecida } \\
\text { principalmente pela comunicação efetiva } \\
\text { em educação em saúde. }\end{array}$ \\
\hline
\end{tabular}

Fonte: Autores (2021). 


\section{Discussão}

Os autores Mesquita et al. (2016) e Sousa et al. (2019) destacam que o leite humano é o alimento adequado para o lactente, nos primeiros seis meses de vida, devido aos benefícios nutricionais e imunológico, além de manter um laço afetivo com a mãe. Estudos científicos apontam o impacto do aleitamento materno na alimentação saudável, na promoção da saúde, prevenção de doenças nutricionais e infecciosas, além disso, oferecendo proteção contra morbimortalidade por doenças infecciosas, nos primeiros anos de vida.

Assim, Viana et al. (2021) define que as dificuldades na amamentação mais frequentemente descritas são ingurgitamento mamário e apojadura dolorosa, escoriações e fissuras, mastites, choro da criança, uso de bicos e chupetas, medicações e drogas, dentre outros. Essas dificuldades devem ser informadas e discutidas com a lactante, porque quase sempre são omitidas e há um enfoque com visão romantizada do ato de amamentar. Mas é sempre possível incentivar o Aleitamento Materno, contornando possíveis problemas e situações transitórias e estimulando a relactação.

Assim, Higashi et al. (2021) fala que em relação à Saúde da Família, o enfermeiro é um profissional obrigatório dentro da equipe básica de Saúde da Família, tem duas funcionalidades ou dois campos essenciais de atuação, na unidade de saúde e na comunidade. Esse profissional tem consigo vários papeis para desempenhar, como o de educador da saúde, prestador de cuidados, planejador, consultor, ouvinte, articulador, entre outros, ele continua sendo o sujeito do seu processo de trabalho, condicionando aos enfermeiros, grande autonomia no exercer da atenção primária à saúde.

Silva et al. (2011) comenta sobre as posições corretas na amamentação, quando o posicionamento da pega da criança é inadequado, deixa os mamilos doloridos, ocasionando desconforto. No processo de apoio à nutriz, é relevante informar que o tamanho do mamilo, por si só, não se constitui em fator que dificulte a amamentação, visto que a parte que o bebê introduz na boca para poder sugar o leite materno representa $1 / 3$ da porção da mama. Já o posicionamento, interfere diretamente na pega e extração do leite, provocando dor e traumas mamilares, como as fissuras. Assim, fica evidente que as dificuldades na amamentação não são isoladas, mas apresentam-se como um conjunto de fatores interligados, confirmando a importância do acompanhamento do profissional de saúde e da implementação da promoção do aleitamento materno.

Sousa et al. (2019) destaca que mesmo sendo considerada uma técnica simples, são necessários alguns cuidados, ao mesmo tempo em que boa dedicação e conhecimento da mãe para realizá-la. Se for executada de forma inapropriada pode acarretar dor e lesão nos mamilos. E nesse momento é que aparece o importante papel do enfermeiro, não somente para orientar a técnica, mas especialmente para promover a autoconfiança da mãe e orientá-la quanto aos fatores que podem favorecer a descida do leite.

As ações de incentivo, promoção e apoio ao aleitamento materno, precisam ocorrer no conjunto das ações dos profissionais, ou seja, durante o pré-natal, o pré-parto, o nascimento, assim como no período de imunizações, teste do pezinho e retorno para a consulta de puerpério, em todas as etapas a enfermagem está presente (Pereira et al., 2021)

A consulta pré-natal deve ser realizada no início da gestação para uma minuciosa avaliação das condições materna e fetal. Embora a gravidez seja um processo fisiológico normal, as várias alterações que ocorrem no corpo da mulher resultam numa menor distância entre saúde e doença do que quando ela não está grávida. A prevenção ou pelo menos diagnóstico precoce dos sinais anormais, seguida por tratamento imediato e efetivo, evitará muitas complicações associadas a parturição, não somente durante o período ante parto, como também durante o trabalho de parto, o nascimento e o puerpério. Portanto, a assistência ante parto é essencialmente uma assistência preventiva para a mãe e o feto (Emídio et al., 2020).

Diante desse contexto, a Rede Cegonha trata-se de uma estratégia do Ministério da Saúde que visa programar uma rede voltada para os cuidados da mulher gestante e assegurar a elas o direito ao planejamento reprodutivo e a atenção humanizada à gravidez, ao parto e ao puerpério, bem como assegurar às crianças o direito ao nascimento seguro e ao crescimento e desenvolvimento saudáveis. Esta estratégia tem a finalidade de estruturar e organizar a atenção à saúde materno- 
infantil no País e será implantada, gradativamente, em todo o território nacional, iniciando sua implantação respeitando o critério epidemiológico, taxa de mortalidade infantil e razão mortalidade materna e densidade populacional (Cunha et al.,2016).

Portanto, a Rede Cegonha desenvolve ações em 5.488 municípios, alcançando 2,6 milhões de gestantes. Desde o lançamento da Rede, foram investidos mais de $\mathrm{R} \$ 3,1$ bilhões para o desenvolvimento das ações. A estratégia também busca contribuir na aceleração da redução das taxas de mortalidade materna e neonatal. Somente em 2013 foram realizadas 18,9 milhões de consultas pré-natais pelo Sistema Único de Saúde (SUS), o que representa aumento de $93 \%$ em relação a 2003 (Lustosa et al., 2020).

Silva et al. (2020) conceitua que a participação precípua dos profissionais de saúde junto às nutrizes no processo do aleitamento materno permite a identificação precoce de práticas que podem prejudicar a amamentação. Dentre os profissionais inseridos na promoção ao aleitamento materno, os que mais se destacam são os enfermeiros, os técnicos de enfermagem e os agentes comunitários de saúde, porém, é sempre importante reforçar a necessidade de participarem de intervenções de capacitação.

Ainda com autor, o enfermeiro deve identificar durante o pré-natal os conhecimentos, a experiência prática, as crenças e a vivência social e familiar da gestante com a finalidade de promover educação em saúde para o aleitamento materno, assim como, garantir vigilância e efetividade durante a assistência a nutriz no pós-parto.

Nos últimos anos, o lançamento de programas prioritários como a Rede Cegonha e o Brasil Carinhoso refletiu a importância com que o governo federal trata as questões voltadas para o público infantil. E os estados e municípios, bem como a sociedade civil organizada, são nossos grandes parceiros na construção de um SUS que investe na infância em que a saúde se encontra, mas a qualidade que a população tem vivido atualmente (Emídio et al., 2020).

O futuro do Brasil tem sido alvo de preocupação por parte dos profissionais de saúde, políticos interessados e gestores públicos, a criança representa um campo de investimento dentro dos cuidados da saúde. Assim, com o passar do tempo, algumas transformações tem sido notadas, no que diz respeito às diretrizes das políticas de saúde voltadas ao público infantil. A mortalidade infantil, por exemplo, reflete não apenas o nível em que a saúde se encontra, mas a qualidade que a população tem vivido atualmente

É importante destacar que o enfermeiro é um profissional que se preocupa com as pessoas, vê não somente a patologia, mas o ser humano como um todo, ajudando o paciente e a família a passar pelos momentos de angustia e dor diante do adoecimento. Contudo, vem evoluindo cientificamente com o passar dos anos, tornando-se referência como cuidador.

\section{Considerações Finais}

A promoção do aleitamento materno ocorre na maioria das vezes na unidade básica de saúde e por isso os profissionais de saúde mais envolvidos nessa prática são aqueles que integram as equipes de saúde da família que geralmente são os médicos, enfermeiros, técnicos de enfermagem e agentes de saúde.

O enfermeiro da equipe de saúde tem um importante papel frente à amamentação, pois são eles quem mais se relaciona com a mulher durante o ciclo gravídico-puerperal, lidando com as demandas do aleitamento, e são por meio de suas práticas que elas podem incentivar a amamentação e apoiar as gestantes, melhorando assim, os índices de aleitamento materno e, consequentemente, diminuindo os índices de desnutrição infantil, alergias, anemias, doenças dentárias e infecções que podem elevar à mortalidade infantil, além de diminuir as internações, custos com consultas, medicamentos e outros.

Por fim, conclui-se que a enfermagem tem grande contribuição quando se trata do apoio para a prática do aleitamento materno e a conscientização sobre o desmame precoce, pois tem conhecimentos práticos e teóricos para oportunizar momentos educativos, orienta gestante e puérperas sobre a importância do Aleitamento Materno e o manejo correto da lactação. 
Com a produção desse trabalho, não somente enfermeiros, mas as mulheres também podem ser alcançadas e uma conscientização sobre a importância do aleitamento materno por período completo por ser feita. Para a comunidade geral, espera-se que estes dados possam ser utilizados na promoção do aleitamento materno, intensificando a prática de amamentar, trazendo benefícios para as famílias e a sociedade, impactando de maneira significativa na redução da mortalidade infantil. Espera-se que as maiorias dos profissionais sejam consciente de suas ações e busquem sempre prestar o melhor apoio a mulher e o recém-nascido e que aspectos relacionados à prestação do serviço de saúde sejam esclarecedores para a promoção do aleitamento materno.

Ainda nesta vertente, cabe-se inferir que existem ainda uma gama de alternativas a serem exploradas por meio de outrosa projetos, tais como a romantizaação da amamentação ou mesmo acerca das ações de promoção de saúde com a população mais vulneraveis, também explorando sobre aleitamento materno nas maternidades.

\section{Referências}

Amaral, L. J. X., Sales, S. D. S., Carvalho, D. P. D. S. R. P., Cruz, G. K. P., Azevedo, I. C. D., \& Ferreira, M. A. (2015). Fatores que influenciam na interrupção do aleitamento materno exclusivo em nutrizes. Revista gaúcha de enfermagem, 36, 127-134.

Cunha, É. C., \& de Siqueira, H. C. H. (2016). Aleitamento materno: contribuições da enfermagem. Ensaios e Ciência: Ciências Biológicas, Agrárias e da Saúde, 20(2), 86-92.

Emidio, S. C. D., Barbosa, F. D., Deberg, J., Moorhead, S., de Souza Oliveira-Kumakura, A. R., \& Carmona, E. V. (2020). Revisão dos indicadores para os Resultados de Enfermagem relacionados ao estabelecimento da amamentação. Revista Eletrônica de Enfermagem, 22 : 1-12.

Fantinato, M. (2015). Métodos de pesquisa. São Paulo: USP.

Galvão, T. F., Pansani, T. D. S. A., \& Harrad, D. (2015). Principais itens para relatar Revisões sistemáticas e Meta-análises: A recomendação PRISMA. Epidemiologia e Serviços de Saúde, 24, 335-342.

Higashi, G. C., dos Santos, S. S., da Silva, R. S., Jantsch, L. B., Soder, R. M., \& da Silva, L. A. A. (2021). Práticas de enfermeiros e a influência sociocultural na adesão ao aleitamento materno. Revista Baiana de Enfermagem35, , e38540.

Lustosa, E., \& Lima, R. N. (2020). Importância da enfermagem frente à assistência primária ao aleitamento materno exclusivo na atenção básica. Revista Brasileira Interdisciplinar de Saúde. 2 (2), 93-7.

Mesquita, A. L., Souza, V. A. B., Santos, T. N. D., \& Santos, O. P. D. (2016). Atribuições de enfermeiros na orientação de lactantes acerca do aleitamento materno. Revista de Divulgação Científica Sena Aires, 5(2), 158-170.

Monteschio, C. A. C., Gaíva, M. A. M., \& Moreira, M. D. D. S. (2015). O enfermeiro frente ao desmame precoce na consulta de enfermagem à criança. Rev Bras Enferm. 68(5); 869-75.

Pereira, A. D. O. R., de Menezes Ferreira, R., de Rezende, F. M., Quadros, K. A. N., dos Santos, R. C., \& Andrade, S. N. (2021). Fatores que interferem na realização do aleitamento materno exclusivo. Nursing (São Paulo), 24(274), 5401-5418.

Paiva, M. R. F., Parente, J. R. F., Brandão, I. R., \& Queiroz, A. H. B. (2016). Metodologias ativas de ensino-aprendizagem: revisão integrativa. SANARERevista de Políticas Públicas, 15(2).

Rocha, F. A. A., Junior, A. R. F., Júnior, C. C. M., \& Rodrigues, M. E. N. G. (2016). O enfermeiro da estratégia de saúde da família como promotor do aleitamento materno. Revista Contexto \& Saúde, 16(31), 15-24.

Ribeiro, O., Martins, M. M., \& Tronchin, D. (2016). Modelos de prática profissional de enfermagem: revisão integrativa da literatura. Revista de Enfermagem Referência, 4(10), 125-134.

Oliveira Fonseca-Machado, M., Parreira, B. D. M., dos Santos Monteiro, J. C., \& Gomes-Sponholz, F. (2015). Perfil sociodemográfico e competência em aleitamento materno dos profissionais de enfermagem da Estratégia Saúde da Família. Revista de Enfermagem Referência, 4(5), 85-92.

Salvador, P. T. C. D. O., Alves, K. Y. A., Rodrigues, C. C. F. M., \& Oliveira, L. V. (2020). Estratégias de coleta de dados online nas pesquisas qualitativas da área da saúde: scoping review. Revista Gaúcha de Enfermagem, 41.

Silva, A. B. L., Alves, B. P., de Sá, B. A., de Souza, J. W. R., de Andrade, M. E., \& Fernandes, M. C. (2021). Experiência e atitudes de gestantes acerca do aleitamento materno. Revista Brasileira em Promoção da Saúde, 4(1): 1-9.

Silva, L. S. D., Leal, N. P. D. R., Pimenta, C. J. L., Silva, C. R. R. D., Frazão, M. C. L. O., \& Almeida, F. D. C. A. D. (2020). Contribuição do enfermeiro ao aleitamento materno na atenção básica. Rev. Pesqui.(Univ. Fed. Estado Rio J., Online),12; 774-778.

Sousa, L. F., de Figueredo, R. C., da Silva Amorim, R. C. C., Silva, L. S., \& Silva, R. S. (2019). Desafios e potencialidades na assistência de enfermagem no aleitamento materno. Revista Remecs-Revista Multidisciplinar de Estudos Científicos em Saúde, 4(7), 17-26. 
Research, Society and Development, v. 10, n. 15, e317101523273, 2021

(CC BY 4.0) | ISSN 2525-3409 | DOI: http://dx.doi.org/10.33448/rsd-v10i15.23273

Viana, M. D. Z. S., da Silveira Donaduzzi, D. S., da Rosa, A. B., \& Fettermann, F. A. Estratégias e ações do enfermeiro no incentivo ao aleitamento materno: revisão integrativa. 13: 1199-1204

Wilhelm, L. A., Demori, C. C., Alves, C. N., Barreto, C. N., Cremonese, L., \& Ressel, L. B. (2015). A vivência da amamentação na ótica de mulheres: contribuições para a enfermagem. Revista de Enfermagem da UFSM, 5(1), 160-168. 